\title{
Influência do teor de sólidos totais na estimativa do coeficiente de dilatação volumétrica do suco de graviola
}

\author{
César Augusto CANCIAM* \\ * Mestre em Engenharia Química, professor lotado no Departamento Acadêmico de Engenharia Química da \\ Universidade Tecnológica Federal do Paraná - Câmpus Ponta Grossa, canciam@utfpr.edu.br
}

Recebido em: 13/03/2014 - Aprovado em: 15/09/2014 - Disponibilizado em: 15/12/2014

\begin{abstract}
RESUMO: Nos líquidos, para se avaliar os impactos no sistema de medição volumétrico decorrentes da variação de temperatura, é necessário fazer um estudo da dilatação volumétrica. O presente trabalho teve por objetivo estudar a dilatação volumétrica do suco de graviola em função do teor de sólidos totais através da estimativa do coeficiente de dilatação volumétrica. Para tanto, realizou-se uma análise de regressão linear de dados estimados da massa específica do suco de graviola com diferentes teores de sólidos totais em função da temperatura. Esses dados foram estimados a partir do modelo empírico proposto por Gratão, Silveira Júnior e Telis-Romero (2007). Para um intervalo de temperatura entre $5^{\circ} \mathrm{C}$ e $65^{\circ} \mathrm{C}$ e teor de sólidos totais entre 10 e $45^{\circ} \mathrm{Brix}$, os valores encontrados para o coeficiente de dilatação térmica foram de $1,96.10^{-4 o} \mathrm{C}^{-1}$ (suco com $45^{\circ} \mathrm{Brix}$ ) a $2,26.10^{-4} \mathrm{C}^{-1}$ (suco com $10^{\circ} \mathrm{Brix}$ ). Em todos os casos estudados, os coeficientes de correlação encontravam-se próximos da unidade, classificando a correlação linear como muito forte. Constatou-se que um aumento no teor de sólidos totais provoca uma diminuição na dilatação volumétrica do suco de graviola.

PALAVRAS-CHAVE: Dilatação volumétrica. Suco. Graviola. Regressão linear. Estimativa.
\end{abstract}

\section{Influence of total solids content in the estimative of the thermal expansion coefficient of soursop juice}

\begin{abstract}
In liquids, to assess the impacts on volumetric measurement system due to temperature variation, it is necessary to make a study of volumetric dilatation. The present work aimed to study the volumetric expansion of soursop juice in function of total solids content by estimating of the thermal expansion coefficient. For this purpose, there was a linear regression analysis of estimate data of the specific weight with diverses total solids content in function of temperature. These values were estimated from the empirical model proposed by Gratão, Silveira Júnior and Telis-Romero (2007). For a temperature range between $5^{\circ} \mathrm{C}$ and $65^{\circ} \mathrm{C}$ and total solids content range between 10 and $45^{\circ} \mathrm{Brix}$, the values obtained for the thermal expansion coefficient were between $1.96 \times 10^{-4} \mathrm{C}^{-1}$ (for juice with $45^{\circ} \mathrm{Brix}$ ) and $2.26 \times 10^{-4}{ }^{\circ} \mathrm{C}^{-1}$ (for juice with $10^{\circ} \mathrm{Brix}$ ). In all cases studied, the correlation coefficients were close to the unit, ranking the linear correlation as very strong. It was found that an increase in total solids content causes a decrease in volumetric dilatation of soursop juice.

KEYWORDS: Volumetric dilatation. Juice. Soursop. Linear regression. Estimate.
\end{abstract}

\section{INTRODUÇÃO}

Com crescente interesse de industrialização, a graviola (Annona muricata L.) apresenta importância do ponto de vista alimentício e farmacológico (SOLÍS-
FUENTES et al., 2010).

Como alimento, a graviola é utilizada na produção de polpas, sucos, néctares, xaropes, geleias, compotas, doces e sorvetes (FASOLIN e CUNHA, 2012; MERCALDI, 2006). 
Países como China, Vietnam, Austrália, Nova Zelândia, Cuba, Bahamas, Granada, México, Costa Rica, Bermudas, República Dominicana, Equador, Colômbia e Brasil destacam-se como produtores dessa fruta (PEÑALOZA, 2008; CARDOZO, 2009).

No Brasil, os estados produtores de graviola são Paraíba, Ceará, Pernambuco e Bahia (MERCALDI, 2006).

O mesmo autor comenta que apesar de sua importância na fruticultura desses estados, não há variedades de graviola que sejam portadoras de características botânicas geneticamente definidas. Isto é, as diversas variedades de graviola se diferenciam pela forma, sabor e consistência de seus frutos. Considerando essas características, no Brasil são conhecidas as variedades Morada, Lisa, Blanca e Crioula.

Segundo Cardozo (2009), a graviola é uma fruta rica em carboidratos e ácidos. Em termos de compostos fitoquímicos presentes na graviola destacam-se alcalóides (como muricina, muricinina, N-metilcoridina e $\mathrm{N}$ metilcorituberina), flavonóides e acetogeninas.

A graviola é constituída, em média, por $54 \%$ de polpa, $36 \%$ de casca e $10 \%$ de sementes. Geralmente ocorre durante o processamento dessa fruta, uma perda de polpa da ordem de $30 \%$ em cada fruta. Essa perda está associada a problemas de caráter agronômico, sendo o mais grave devido à presença de broca dos frutos (NAZARÉ, 2000).

Abbo, Olurin e Odeyemi (2006) relatam que o suco de graviola é composto de água $(87,5 \%)$, carboidratos $(11,3 \%)$, proteínas $(0,64 \%)$, minerais $(0,51 \%)$ e lipídios $(0,05 \%)$.

Os materiais tendem a reagir diferentemente a uma variação da temperatura. Esse comportamento está relacionado com a proximidade e interação entre suas espécies químicas e consequentemente ao estado físico do material. Assim, líquidos e gases tendem a interagir de forma distinta aos sólidos e entre si também são distintos (CANCIAM, 2013a).

O estudo da dilatação térmica em líquidos é feito somente em relação à dilatação volumétrica, sendo importante o conhecimento do coeficiente de dilatação volumétrica (JERÔNIMO, BALBINO e FERNANDES, 2012).

De acordo com Canciam (2013a), o conhecimento do coeficiente de dilatação volumétrica permite estudar o comportamento da dilatação de um material, avaliar os impactos no sistema de medição volumétrico em virtude da variação da temperatura, servir como dado em projetos de equipamentos e acessórios, considerando as dilatações resultantes das fortes variações de 
temperatura; estimar outras propriedades como tensão volumétrica e entalpia de vaporização, estudar a convecção livre e sua influência na transferência de calor em tubulações.

Os valores do coeficiente de dilatação volumétrica estão relacionados com a energia de ligação química entre as espécies. De maneira que, materiais em que as ligações químicas são fortes apresentam baixos coeficientes de dilatação térmica (CANCIAM, 2013a).

A dilatação térmica está associada à variação assimétrica da energia de ligação com a distância entre as espécies químicas. À medida que ocorre o aquecimento do material, as espécies químicas aumentam a frequência e a amplitude de vibração. Como as forças de repulsão são sempre maiores que as forças de atração, a distância entre as espécies químicas também aumenta (SANTOS e VIEIRA, 2010).

O objetivo deste trabalho foi estudar a dilatação volumétrica do suco de graviola com diferentes teores de sólidos totais através da estimativa do coeficiente de dilatação volumétrica. Para tanto, foi realizada uma análise de regressão linear de dados estimados da massa específica do suco de graviola com diferentes teores de sólidos totais em função da temperatura.

\section{MATERIAIS E MÉTODOS}

O cálculo do coeficiente de dilatação volumétrica $(\beta)$ seguiu a metodologia proposta por Canciam (2012).

Essa metodologia considera que o coeficiente de dilatação volumétrica é numericamente igual ao coeficiente angular da reta obtida pelo gráfico de $\ln \left(\frac{\rho_{0}}{\rho}\right)$ versus $\left(T-T_{0}\right)$. Em que $\rho$ corresponde à massa específica na temperatura $T$ e $\rho_{0}$ corresponde à massa específica na temperatura inicial $T_{0}$.

$\mathrm{Na}$ obtenção da massa específica do suco de graviola em função da temperatura utilizou-se a modelagem empírica proposta por Gratão, Silveira Júnior e Telis-Romero (2007).

Esses autores estudaram o comportamento da massa específica do suco de graviola entre $0,4^{\circ} \mathrm{C}$ e $68,8^{\circ} \mathrm{C}$ e teores de sólidos totais variando de $9,3^{\circ} \mathrm{Brix}$ a 49,4 $4^{\circ}$ Brix. A Equação 1 indica a modelagem empírica encontrada por esses autores.

$$
\rho=981,4+4,5 \cdot C+0,23 \cdot T
$$

Em que $C$ corresponde ao teor de sólidos totais do suco de graviola (em $\left.{ }^{\circ} \mathrm{Brix}\right)$ e 
$T$, à temperatura $\left(\mathrm{em}^{\circ} \mathrm{C}\right)$. Na Equação 1 , a unidade para a massa específica $(\rho)$ é kg.m ${ }^{-3}$.

A partir da Equação 1, foram estimados os valores da massa específica do suco de graviola para temperaturas entre 5 e $65^{\circ} \mathrm{C}$ e teores de sólidos totais variando de 10 a $45^{\circ}$ Brix.

A Tabela 1 relaciona os valores estimados da massa específica do suco de graviola nos teores de sólidos totais de 10, 15, 20 e $25^{\circ}$ Brix. Enquanto que a Tabela 2 relaciona os valores estimados da massa específica do suco de graviola nos teores de sólidos totais de $30,35,40$ e $45^{\circ}$ Brix.

Tabela 1. Valores estimados da massa específica do suco de graviola nos teores de sólidos totais de 10, 15,

\begin{tabular}{ccccc}
\multicolumn{5}{c}{20 e $25^{\circ}$ Brix. } \\
\hline $\mathrm{T}\left({ }^{\circ} \mathrm{C}\right)$ & $10^{\circ}$ Brix & $15^{\circ}$ Brix & $20^{\circ}$ Brix & $25^{\circ}$ Brix \\
\hline 5 & 1025,25 & 1047,75 & 1070,25 & 1092,75 \\
10 & 1024,10 & 1046,60 & 1069,10 & 1091,60 \\
15 & 1022,95 & 1045,45 & 1067,95 & 1090,45 \\
20 & 1021,80 & 1044,30 & 1066,80 & 1089,30 \\
25 & 1020,65 & 1043,15 & 1065,65 & 1088,15 \\
30 & 1019,50 & 1042,00 & 1064,50 & 1087,00 \\
35 & 1018,35 & 1040,85 & 1063,35 & 1085,85 \\
40 & 1017,20 & 1039,70 & 1062,20 & 1084,70 \\
45 & 1016,05 & 1038,55 & 1061,05 & 1083,55 \\
50 & 1014,90 & 1037,40 & 1059,90 & 1082,40 \\
55 & 1013,75 & 1036,25 & 1058,75 & 1081,25 \\
60 & 1012,60 & 1035,10 & 1057,60 & 1080,10 \\
65 & 1011,45 & 1033,95 & 1056,45 & 1078,95 \\
\hline
\end{tabular}

Fonte: Autor.

Tabela 2. Valores estimados da massa específica do suco de graviola nos teores de sólidos totais de 30,35 , 40 e $45^{\circ}$ Brix.

\begin{tabular}{ccccc}
\hline $\mathrm{T}\left({ }^{\circ} \mathrm{C}\right)$ & $30^{\circ}$ Brix & $35^{\circ}$ Brix & $40^{\circ}$ Brix & $45^{\circ}$ Brix \\
\hline 5 & 1115,25 & 1137,75 & 1160,25 & 1182,75 \\
10 & 1114,10 & 1136,60 & 1159,10 & 1181,60 \\
15 & 1112,95 & 1135,45 & 1157,95 & 1180,45 \\
20 & 1111,80 & 1134,30 & 1156,80 & 1179,30 \\
25 & 1110,65 & 1133,15 & 1155,65 & 1178,15 \\
30 & 1109,50 & 1132,00 & 1154,50 & 1177,00
\end{tabular}

\begin{tabular}{lllll}
35 & 1108,35 & 1130,85 & 1153,35 & 1175,85 \\
40 & 1107,20 & 1129,70 & 1152,20 & 1174,70 \\
45 & 1106,05 & 1128,55 & 1151,05 & 1173,55 \\
50 & 1104,90 & 1127,40 & 1149,90 & 1172,40 \\
55 & 1103,75 & 1126,25 & 1148,75 & 1171,25 \\
60 & 1102,60 & 1125,10 & 1147,60 & 1170,10 \\
65 & 1101,45 & 1123,95 & 1146,45 & 1168,95 \\
\hline
\end{tabular}
Fonte: Autor.

A partir dos valores indicados nas Tabelas 1 e 2, aplicando a metodologia proposta por Canciam (2012), foram obtidos o coeficiente de dilatação volumétrica $(\beta)$ e o coeficiente de correlação $\left(R^{2}\right)$.

Nos cálculos para a obtenção dos coeficientes de dilatação volumétrica e de correlação, considerou-se como temperatura inicial $\left(T_{0}\right)$ o valor de $5^{\circ} \mathrm{C}$ e a massa específica inicial $\left(\rho_{0}\right)$, à massa específica referente a essa temperatura para cada suco de graviola estudado.

\section{RESULTADOS E DISCUSSÃO}

As Figuras 1 e 2 ilustram os gráficos de $\ln \left(\frac{\rho_{0}}{\rho}\right)$ versus $\left(T-T_{0}\right)$ para cada um dos sucos de graviola estudados.

Enquanto que a Tabela 3 relaciona os resultados obtidos para os coeficientes de dilatação volumétrica $(\beta)$ e de correlação $\left(R^{2}\right)$ 
Figura 1: Gráfico de $\ln \left(\frac{\rho_{0}}{p}\right)$ versus $\left(T-T_{0}\right)$ para teores de sólidos totais entre 10 e $25^{\circ} \mathrm{Brix}$.

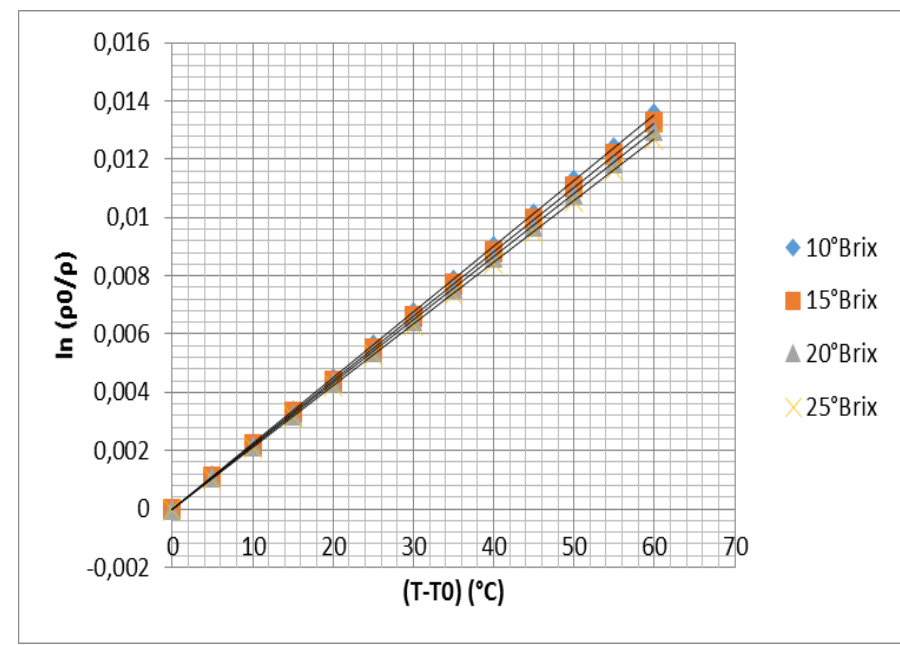

Fonte: Autor.

Figura 2: Gráfico de $\ln \left(\frac{\rho_{0}}{\rho}\right)$ versus $\left(T-T_{0}\right)$ para teores de sólidos totais entre 30 e $45^{\circ}$ Brix.

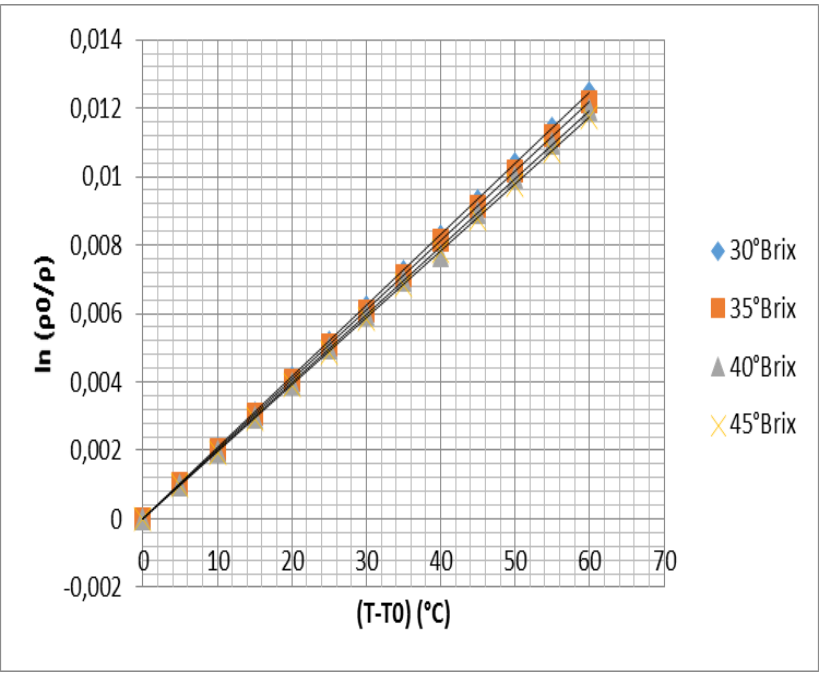

Fonte: Autor.

Tabela 3. Valores estimados do coeficiente de dilatação volumétrica e do coeficiente de correlação.

\begin{tabular}{ccc}
\hline $\mathrm{C}\left({ }^{\circ}\right.$ Brix $)$ & $\beta\left({ }^{\circ} \mathrm{C}^{-1}\right)$ & $R^{2}$ \\
\hline 10 & $2,26.10^{-4}$ & 0,99 \\
15 & $2,21.10^{-4}$ & 0,99 \\
20 & $2,16.10^{-4}$ & 0,99 \\
25 & $2,12.10^{-4}$ & 0,99 \\
30 & $2,08.10^{-4}$ & 0,99
\end{tabular}

\begin{tabular}{rrr}
35 & $2,03.10^{-4}$ & 0,99 \\
40 & $1,99.10^{-4}$ & 0,99 \\
45 & $1,96.10^{-4}$ & 0,99 \\
\hline
\end{tabular}

Fonte: Autor.

Foi realizada uma pesquisa na literatura a fim de obter valores do coeficiente de dilatação volumétrica do suco de graviola e constatou-se a ausência de valores experimentais para essa grandeza. Dessa forma, dificulta a análise dos valores obtidos neste trabalho.

De acordo com Cabral e Lago (2002), o coeficiente de dilatação volumétrica da água equivale a $2,07 \cdot 10^{-4} \mathrm{C}^{-1}$.

A molécula de água é uma molécula polar. A interação entre suas moléculas é do tipo ligação de hidrogênio. A ligação de hidrogênio ocorre quando um átomo de hidrogênio liga-se por covalência a um átomo mais eletronegativo e mantém uma afinidade residual por outro átomo eletronegativo, apresentando uma tendência à carga positiva (BROWN e HOLME, 2009).

O teor de sólidos totais (medidos em ${ }^{\circ}$ Brix) indica a quantidade de substâncias dissolvidas no suco, sendo constituídas, em sua maioria, por açúcares (glicose, frutose e sacarose) (CHAVES et al., 2004).

Moléculas de glicose, frutose e sacarose apresentam em sua estrutura grupamentos hidroxila, que são polares. No estado sólido, as moléculas desses açúcares encontram-se ligadas umas às outras através 
das ligações de hidrogênio. Quando ocorre a dissolução desses açúcares na água, são estabelecidas novas ligações de hidrogênio entre os grupamentos hidroxila das moléculas desses açúcares e as moléculas de água, o que garante a dissolução desses açúcares na água. Entretanto, também são estabelecidas forças intermoleculares do tipo dipolo permanentedipolo permanente entre as moléculas desses açúcares e da água (PERUZZO e CANTO, 2010).

As ligações de hidrogênio, quando comparadas com as forças intermoleculares do tipo dipolo permanente-dipolo permanente, são mais fortes (BROWN e HOLME, 2009).

Comparando os coeficientes de dilatação volumétrica da água e dos sucos de graviola estudados, percebe-se que o coeficiente de dilatação volumétrica da água é menor que o coeficiente de dilatação térmica dos sucos de graviola com 10, 15, 20, 25 e $30^{\circ}$ Brix. Provavelmente nos sucos de graviola com esses teores de sólidos totais prevaleçam as interações intermoleculares do tipo dipolo permanente-dipolo permanente. E para sucos de graviola com teores de sólidos totais de 35 , 40 e $45^{\circ}$ Brix, prevalecem as ligações de hidrogênio formadas entre as moléculas de água e dos açúcares dissolvidos.

Todas essas considerações necessitam ser mais estudadas e discutidas.
Com relação ao coeficiente de correlação, Pinheiros e coautores (2009) comentam que esse parâmetro mede a interdependência linear entre as variáveis e avalia a qualidade do ajuste, ou seja, quanto mais próximo o coeficiente de correlação for da unidade, melhor o ajuste da reta em relação aos pontos da dispersão.

Lira (2004) fornece uma classificação para as correlações lineares. A autora comenta que a correlação linear é classificada como muito forte quando os módulos dos valores do coeficiente de correlação são maiores ou iguais a 0,90 e menores que 1,0 .

Assim, pode-se observar na Tabela 3 , que para os sucos de graviola estudados, a correlação linear é classificada como muito forte.

A Figura 3 ilustra o gráfico do coeficiente de dilatação volumétrica do suco de graviola em função do teor de sólidos totais.

Pode-se na Figura 3 que na medida em que o teor de sólidos totais do suco de graviola aumenta ocorre uma diminuição no coeficiente de dilatação volumétrica. E essa diminuição é de maneira linear. 
Figura 3: Gráfico do coeficiente de dilatação volumétrica em função do teor de sólidos totais.

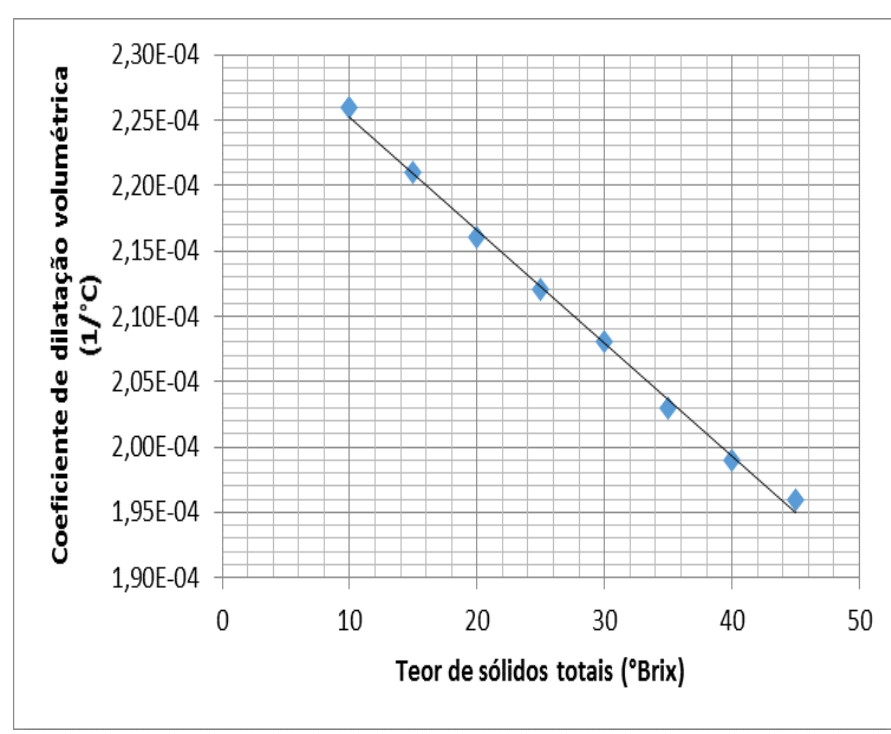

Fonte: Autor.

A Equação 2 corresponde à equação da reta obtida pela análise de regressão linear dos dados do coeficiente de dilatação volumétrica em função do teor de sólidos totais.

$$
\rho=-8,6429 \cdot 10^{-7} \cdot C+2,3389 \cdot 10^{-4}
$$

O coeficiente de correlação para essa análise equivale a -0,99.

Dessa forma, os resultados sugerem que a correlação entre o coeficiente de dilatação volumétrica do suco de graviola e o teor de sólidos totais é uma correlação linear, classificada como muito forte.

Zuritz e coautores (2005) estudaram a influência do teor de sólidos totais e da temperatura no coeficiente de dilatação volumétrica de sucos clarificados de uva e determinaram uma expressão empírica polinomial de grau 2 tanto para a temperatura como para o teor de sólidos totais.

Já Canciam (2013b), estudando a influência do teor de sólidos totais no coeficiente de dilatação volumétrica de sucos de abacaxi, observou que existe uma correlação linear entre o coeficiente de dilatação volumétrica e o teor de sólidos totais e que à medida em que o teor de sólidos aumenta, ocorre uma diminuição nos valores do coeficiente de dilatação volumétrica.

De acordo Santos e Vieira (2010), a partir do conhecimento do coeficiente de dilatação volumétrica é possível avaliar os impactos no sistema de medição volumétrico.

Neste sentido, considerando um volume de 1000 litros de água com uma variação de temperatura de $10^{\circ} \mathrm{C}$, o volume de água aumenta em torno de 2,07 litros. Com esse mesmo raciocínio, o volume de 1000 litros de suco de graviola com $10^{\circ}$ Brix, com uma variação de temperatura de $10^{\circ} \mathrm{C}$, o volume desse suco aumenta em torno de 2,26 litros.

A Tabela 4 relaciona a ordem de aumento de volume para uma variação de temperatura de $10^{\circ} \mathrm{C}$, considerando um volume de 1000 litros para cada um dos sucos de graviola estudados. 
Tabela 4. Ordem de aumento de volume em função do teor de sólidos totais.

\begin{tabular}{cc}
\hline $\mathrm{C}\left({ }^{\circ}\right.$ Brix $)$ & Ordem de aumento $(\mathrm{L})$ \\
\hline 10 & 2,26 \\
15 & 2,21 \\
20 & 2,16 \\
25 & 2,12 \\
30 & 2,08 \\
35 & 2,03 \\
40 & 1,99 \\
45 & 1,96 \\
\hline
\end{tabular}

Fonte: Autor.

Pode-se observar na Tabela 4 que na medida em que se aumenta o teor de sólidos totais do suco de graviola, menor é a ordem de aumento de volume, em virtude dos valores do coeficiente de dilatação volumétrica.

\section{CONCLUSÃO}

O objetivo deste trabalho foi estudar a dilatação volumétrica do suco de graviola com diferentes teores de sólidos totais através da estimativa do coeficiente de dilatação volumétrica. Para tanto, foi realizada uma análise de regressão linear de dados estimados da massa específica do suco de graviola com diferentes teores de sólidos totais em função da temperatura.

Para um intervalo de temperatura entre 5 e $65^{\circ} \mathrm{C}$ e teor de sólidos totais entre 10 e $45^{\circ}$ Brix, os valores encontrados para o coeficiente de dilatação volumétrica variaram de $1,96.10^{-4}$ a $2,26.10^{-4} \mathrm{C}^{-1}$.

Em todos os casos estudados, os coeficientes de correlação encontravam-se próximos da unidade, classificando a correlação linear como muito forte.

Constatou-se que um aumento no teor de sólidos totais provoca uma diminuição na dilatação volumétrica do suco de graviola. A justificativa para tal comportamento pode estar associada às interações intermoleculares estabelecidas entre as espécies químicas encontradas no suco de graviola.

Os resultados sugerem que na medida em que se aumenta o teor de sólidos totais do suco de graviola, menor é a ordem de aumento de volume, em virtude dos valores do coeficiente de dilatação térmica.

\section{REFERÊNCIAS}

ABBO, E. S.; OLURIN, T. O.; ODEYEMI, G. Studies on the storage stability of soursop (Annona muricata L.) juice. African Journal of Biotechnology, v. 5, n. 19, p. 1808-1812, 2006.

BROWN, L. S.; HOLME, T. A. Química geral aplicada à Engenharia. Cengage Learning, São Paulo, 2009. 653p.

CABRAL, F.; LAGO, A. Física 2. Harbra, São Paulo, 2002. 516p.

CANCIAM, C. A. Predição do coeficiente de expansão térmica do óleo de girassol através da aplicação da análise de regressão linear. Revista Brasileira de Tecnologia Agroindustrial, v. 6, n. 2, p. 852-863, 2012.

CANCIAM, C. A. Estimativa do coeficiente de expansão térmica de misturas de óleo diesel e óleo de soja residual. Revista E-xacta, v. 6, n. 1, p. 67-74, 2013a.

CANCIAM, C. A. Correlação entre o coeficiente de expansão térmica e o teor de 
sólidos totais para o suco de abacaxi. Revista Brasileira de Tecnologia Agroindustrial, v. 7, n. 2, p. 1128-1139, 2013 b.

CARDOZO, C. J. M. Caracterización fisiológica, físico-química, reológica, nutracéutica, estructural y sensorial de la guanábana (Annona muricata L. cv. ELITA). Tese de Doutorado em Ciências da Universidad Nacional de Colombia, 2009. 274p.

CHAVES, M. C. V.; GOUVEIRA, J. P. G.; ALMEIDA, F. A. C.; LEITE, J. C. A.; SILVA, F. L. H. Características físico-químicas do suco de acerola. Revista de Biologia e Ciências da Terra, v. 4, n. 2, p. 1-10, 2004.

FASOLIN, L. H.; CUNHA, R. L. Soursop juice stabilized with soy fractions: a rheological approach. Revista Ciência e Tecnologia de Alimentos, v. 32, n. 3, p. 558-567, 2012.

GRATÃO, A. C. A.; SILVEIRA JÚNIOR, V.; TELIS-ROMERO, J. Laminar flow of soursop juice through concentric annuli: friction factors and rheology. Journal of Food Engineering, v. 78, p. 1343-1354, 2007.

JERÔNIMO, C. E. M.; BALBINO, C. P.; FERNANDES, H. G. Coeficiente de dilatação volumétrica determinados pela curva ASTM em frações de petróleo. Revista Scientia Plena, v. 8, n. 9, p. 1-8, 2012.

LIRA, S. A. Análise de correlação: abordagem teórica e de construção dos coeficientes com aplicação. Dissertação de Mestrado do Programa de Mestrado em Ciências da Universidade Federal do Paraná, 2004. 196p.

MERCALDI, J. C. Desenvolvimento de bebida à base de "leite" de soja acrescida de suco de graviola. Dissertação de Mestrado em Ciências dos Alimentos da Universidade Estadual Paulista "Júlio de Mesquita Filho", 2006. 61p.

NAZARÉ, R. F. R. Produtos agroindustriais de bacuri, cupuaçu, graviola e açaí desenvolvidos pela Embrapa Amazônica Oriental. Embrapa,
Belém, 2000. 27p.

PEÑALOZA, A. M. C. Estudio comparativo de três sistemas de secado para la producción de um polvo deshidratado de fruta. Dissertação de Mestrado em Engenharia Química da Universidad Nacional de Colombia, 2008. 111p.

PERUZZO, F. M.; CANTO, E. L. Química na abordagem do cotidiano - volume 1: Química Geral e Inorgânica. Moderna, São Paulo, 2010. 648p.

PINHEIRO, J. I. D.; CUNHA, S. B.; CARVAJAL, S. R.; GOMES, G. C. Estatística básica: a arte de trabalhar com dados. Elsevier, Rio de Janeiro, 2009. 288p.

SANTOS, D. Q.; VIEIRA, D. F. Determinação de coeficiente de expansão térmica do biodiesel e seus impactos no sistema de medição volumétrico. Revista Eclética Química, v. 35, n. 4, p. 107-112, 2010.

SOLÍS-FUENTES, J. A.; AMADORHERNÁNDEZ, C.; HERNÁNDEZ-MEDEL, R.; DURÁN-DE-BAZÚA, M. C.

Caracterización fisicoquímica y comportamento térmico del aceite de "almendra" de guanábana (Annona muricata, L.). Grasas y Aceites, v. 61, n. 1, p. 58-66, 2010.

ZURITZ, C. A.; PUNTES, E. M.; MATHEY, H. H.; PÉREZ, E. H.; GASCÓN, A.; RUBIO, L. A.; CARULLO, C. A.; CHERNIKOFF, R. E.; CABEZA, M. S. Density, viscosity and coefficient of thermal expansion of clear grape juice at different solubles solid concentrations and temperatures. Journal of Food Engineering, v. 71, p. 143-149, 2005. 